\title{
The magnetic observatory on Tatuoca, Belém, Brazil: history and recent developments
}

\author{
Achim Morschhauser ${ }^{1}$, Gabriel Brando Soares ${ }^{2}$, Jürgen Haseloff ${ }^{1}$, Oliver Bronkalla $^{1}$, José Protásio ${ }^{2}$, Katia Pinheiro ${ }^{2}$, \\ and Jürgen Matzka ${ }^{1}$ \\ ${ }^{1}$ GFZ German Research Centre for Geosciences, Geomagnetism, Telegrafenberg, 14473 Potsdam, Germany \\ ${ }^{2}$ Geophysics Department, Observatório Nacional, Rio de Janeiro, CEP, 20921-400, Brazil \\ Correspondence to: Achim Morschhauser (mors@gfz-potsdam.de)
}

Received: 3 March 2017 - Discussion started: 9 March 2017

Revised: 18 June 2017 - Accepted: 27 June 2017 - Published: 10 October 2017

\begin{abstract}
The Tatuoca magnetic observatory (IAGA code: TTB) is located on a small island in the Amazonian delta in the state of Pará, Brazil. Its location close to the geomagnetic equator and within the South Atlantic Anomaly offers a high scientific return of the observatory's data. A joint effort by the National Observatory of Brazil (ON) and the GFZ German Research Centre for Geosciences (GFZ) was undertaken, starting from 2015 in order to modernise the observatory with the goal of joining the INTERMAGNET network and to provide real-time data access. In this paper, we will describe the history of the observatory, recent improvements, and plans for the near future. In addition, we will give some comments on absolute observations of the geomagnetic field near the geomagnetic equator.
\end{abstract}

\section{Introduction}

The Tatuoca magnetic observatory (IAGA code: TTB) has a long history, and its roots go back to as early as 1933 when a temporal magnetic observatory was set up on the island of Tatuoca (Gama, 1955). Already at that time, the site of the observatory was chosen to fall within low magnetic latitudes (Gama, 1955), and an inclination of $18.18^{\circ}$ was measured when a permanent magnetic observatory was opened on Tatuoca in 1954 (AGU, 1955). Eventually, the northwardmoving equator passed the observatory in March 2013 (Fig. 1). The closest neighbouring observatory of Tatuoca is located in Kourou (IAGA code: KOU, French Guyana) at a distance of about $700 \mathrm{~km}$ north and $400 \mathrm{~km}$ west of Tatuoca (Fig. 1), and was installed in 1995 by the Institut de Physique du Globe de Paris (IPGP). While the TTB observatory is currently under the full influence of the equatorial electrojet (EEJ), the KOU observatory is far enough from the magnetic equator to record this signal. Thus, subtracting the magnetic data recorded at the KOU observatory from those recorded at the TTB observatory will isolate the magnetic signal of the EEJ from the signal of the solar quiet (SQ) currents and the magnetospheric ring currents (cf. Manoj et al., 2006).

Moreover, the Tatuoca observatory is located within the South Atlantic Anomaly (SAMA) (Hulot et al., 2015), and shows a strong secular variation of almost $200 \mathrm{nT} \mathrm{yr}^{-1}$ in the radial component as predicted, for example, by IGRF-12 (Thébault et al., 2015).

The National Observatory of Brazil (ON) and the German Research Centre for Geosciences (GFZ) are currently preparing the Tatuoca observatory to join the INTERMAGNET network. This will add a third observatory to the INTERMAGNET equatorial observatories, besides Huancayo and Addis Ababa. In 2015 and 2016, two trips were organised to Tatuoca in order to equip the observatory with modern instrumentation, to train the local observers in a different type of absolute measurement, and to update data processing routines. Also, real-time data become more important with respect to applications for space weather monitoring and directional drilling (Buchanan et al., 2013), and a long-term goal of this project is to provide real-time data of the TTB observatory.

In this paper, we will first give an overview of the observatory location and its infrastructure. Then, we will summarise the history of the observatory, with a focus on the technical and operational state before the initiation of this project. 


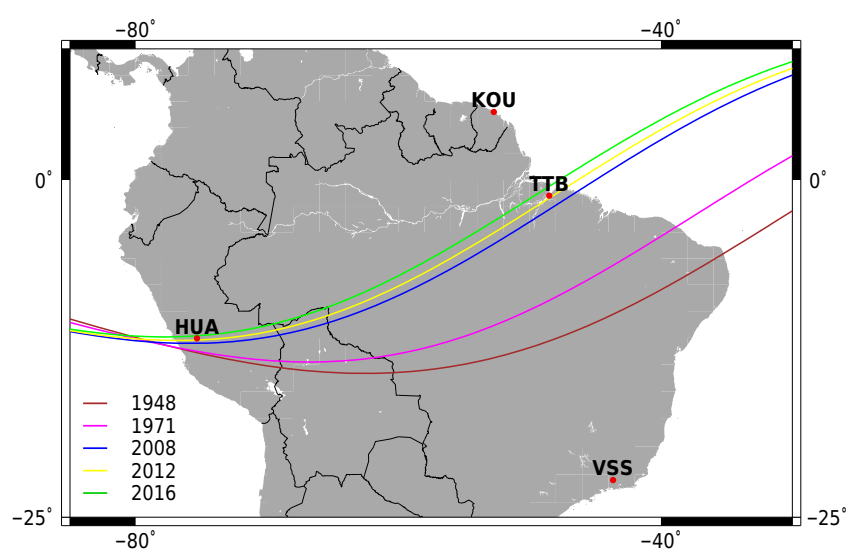

Figure 1. The location of the Tatuoca (TTB) observatory is shown along with INTERMAGNET observatories in the region (Kourou (KOU), Huancayo (HUA), and Vassouras (VSS)). In addition, the location of the geomagnetic equator according to IGRF is shown for different years between 1948 and 2016, as indicated by the colours.

This summary is followed by a description of the recent improvements and the current state of the observatory before we shortly comment on the data and their availability. The paper concludes with a summary and outlook.

\section{History of the observatory}

The history of the Tatuoca magnetic observatory started in 1925 when the ON considered installing a permanent station within the equatorial region. This goal was reinforced by a recommendation of the International Union of Geodesy and Geophysics (IUGG) in 1933, after an Assembly in Lisboa. At this date, two La Cour type magnetographs were provided by the International Polar Year Commission to the National Observatory of Brazil (Gama, 1955). Subsequently, Mr Marquez $(\mathrm{ON})$ was in charge of finding a location free from artificial magnetic disturbances, and he chose a small island owned by the Brazilian government and close to the city of Belém. The magnetic station on Tatuoca operated only from September 1933 to January 1934 due to lack of funding. Important results from these recordings were published in 1951 by the Temporary Commission on the Liquidation of the Polar Year 1933-1934 (Olsen, 1951; IUGG, IPY, 1950). In the subsequent years, the Tatuoca project had been halted due to budget limitations, especially during World War II (Gama, 1955).

In 1951, UNESCO offered a Ruska field theodolite magnetometer inductor for absolute measurements to the $\mathrm{ON}$, with the condition that the Brazilian government will finance the necessary buildings. The construction of the variometer and absolute houses on Tatuoca was completed in 1953, and the office and other buildings were ready in 1954. In this year, a magnetograph was supplied by the Inter American Geodetic Survey which was installed by W. C. Parkinson (Depart- ment of Terrestrial Magnetism, Carnegie Institution of Washington) and by L. I. Gama, the director of ON (AGU, 1955).

Many tests on the absolute and variometer measurements were performed to check the feasibility of a magnetic observatory on Tatuoca. However, due to logistic problems, this was achieved only in 1957 when J. Kozlosky (Inter American Geodetic Survey) visited the island for a few days. On 19 August 1957, the Tatuoca magnetic observatory started its regular operation, and is also listed as a station of the International Geophysical Year 1957-1958 (Nicolet and Doyen, 1959). The observatory has been providing continuous data except for data gaps between 1979 and 1980 due to technical problems and renovation work (Ferreira, 1990). Hourly mean data from Tatuoca were published each year in internal reports of ON. In addition, most hourly mean values from 1957 to 1959,1964 to 1965 , and from 1990 to 1999 are published at the World Data Centre Edinburgh (WDC). During most of this time (1970s to 1990s), José Teotônio Ferreira was responsible for data processing at the observatory. As well, Luiz Muniz Barreto, who was responsible for the Tatuoca and Vassouras observatories during six decades, and director of ON in the 1970s and 1980s, needs to be mentioned here.

In May 1996, a digital automatic station was installed in Tatuoca and the classical variometers were disabled. However, the digital station presented problems in February 1997 and the classical variometers were reactivated (Ferreira, 1998). Finally, the last magnetogram from the classical variometers on Tatuoca was obtained on 13 May 2007. The classical variometers stopped working due to the lack of photograph paper, which was out of production. As a substitute, a LEMI 417 variometer was installed for continuous vector measurements. Also, a POS-1 scalar magnetometer was installed in 2007, which stopped working in 2013 due to insufficient power supply.

\section{Observatory location and set-up}

The Tatuoca magnetic observatory is located at $1.205^{\circ} \mathrm{S}$ and $311.487^{\circ} \mathrm{E}$ (geodetic coordinates) on a small island within the Amazon Delta in the state of Pará in Brazil, and the island is located a $1 \mathrm{~h}$ boat trip away from the port of Icoaraci close to the city of Belém (Figs. 1 and 2). Further, the island of Tatuoca has an approximate size of $460 \mathrm{~m}$ by $300 \mathrm{~m}$, and is largely covered by dense vegetation (Fig. 3). A big advantage is that the island is exclusively used by the observatory and owned by the Brazilian government. Therefore, it is well protected from any artificial disturbances, and had never been relocated during its 65 years of existence.

On the island of Tatuoca, several buildings are located which are related to the observatory (Fig. 3). From south to north, there is a residential house for the observatory staff, an electronics house with the batteries and solar regulators as well as a small office and a storage house with diesel gener- 


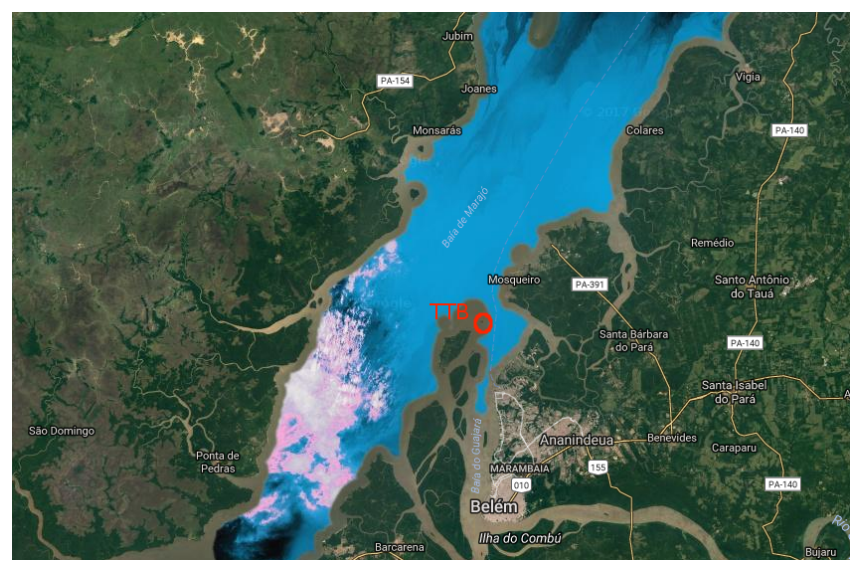

Figure 2. Satellite image of Tatuoca island and its surroundings (Copyright 2017 Landsat/Copernicus, TerraMetrics and Google). The city of Belém is located to the south of Tatuoca island and the observatory.

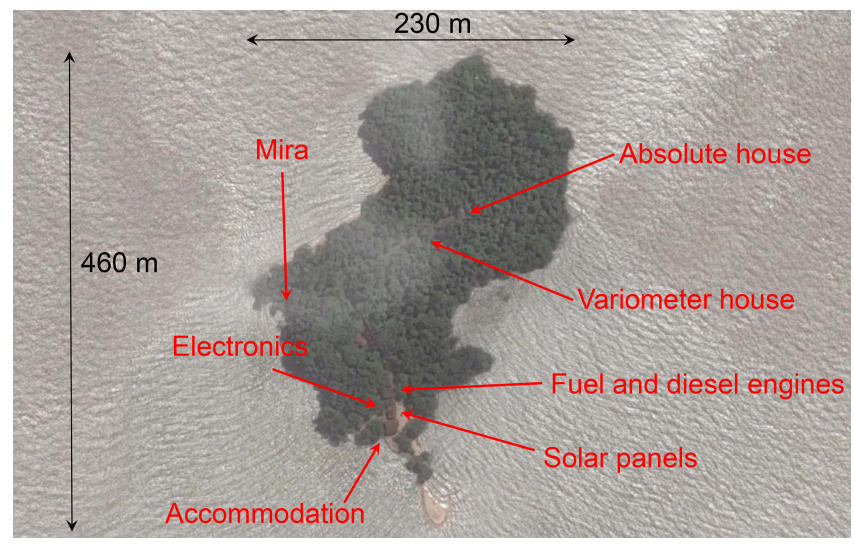

Figure 3. Satellite image of Tatuoca island (copyright 2016 Google). The main buildings and infrastructure of the observatory are marked and annotated.

ators. In the north-eastern corner of the island, the absolute and variometer houses are located (Fig. 4).

A schematic drawing of the variometer house is shown in Fig. 5. The variometer house consists of an outer corridor (light grey) and an inner insulated room (dark grey). The insulation dampens the temperature variation to between 30 and $35^{\circ} \mathrm{C}$ (in 2016), with a maximum daily variation of $3 \mathrm{~K}$. The inner room is equipped with two large solid pillars and one smaller pillar in the south-eastern corner (Fig. 5, all shown in black). In addition, two wooden shelves are located in the southern part of the inner room (black-grey checked). On the easternmost of the solid pillars, a LEMI-417 vector fluxgate magnetometer is located (yellow circle), and the electronics of this instrument is located near the entrance of the variometer house (yellow rectangle). The LEMI system is powered by a $45 \mathrm{Ah}$ lead-acid battery which is charged by a dedicated $30 \mathrm{~W}$ solar panel on the roof of the variometer

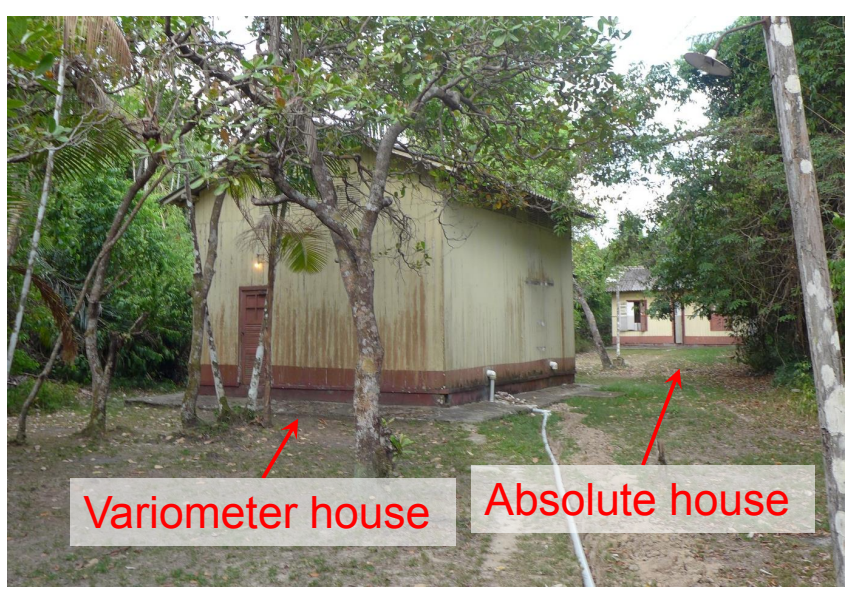

Figure 4. The variometer and absolute houses of Tatuoca observatory are shown. These are located at the north-eastern corner of the island (Fig. 3).

house. Further, a POS-2 proton gradiometer was located in the south-eastern corner of the variometer house (shown in white, Fig. 5). This instrument was never in operation and was removed in October 2016 (see below).

The absolute house has an approximate size of 4.8 by $8.0 \mathrm{~m}$, and is roughly oriented in $\mathrm{N}-\mathrm{S}$ direction. It houses 10 pillars, 4 of which are located at the northern end, including the main pillar, and 6 of which are located at the southern end. The latter pillars carry several historic instruments, including the Ruska theodolite donated by UNESCO. The main pillar is equipped with a ZEISS 020B theodolite in degree scale to which a Canadian EDA fluxgate magnetometer had been attached. The EDA fluxgate had an analogue current reading, and therefore the absolute measurements had to be performed with the zero residual method (Newitt et al., 1996, p. 43ff). As described in Sect. 4, the fluxgate has been replaced with a digital instrument during our first trip in November 2015. For absolute measurements, an azimuth mark is located at a distance of $150 \mathrm{~m}$ to the southwest. Further, a GEM System GSM-19 proton Overhauser magnetometer is available for measuring the magnetic field intensity. Until recently, the time of the absolute measurements was taken from an analogue wall clock which is regularly set according to the GPS time of the LEMI electronics in the variometer house.

In total, there are three observers and one cook who swap shifts in teams of two each week. Therefore, the observatory is usually occupied by two persons who do two consecutive absolute measurements on 3 days each week. In addition, the head of the observatory and one technician are both located in Belém, and frequently visit the island. For this purpose, and for transporting goods and fuel to the island, the observatory owns a small motorboat.

Concerning power supply, the observatory is equipped with recently upgraded solar panels of nominal $324 \mathrm{~W}$ to- 


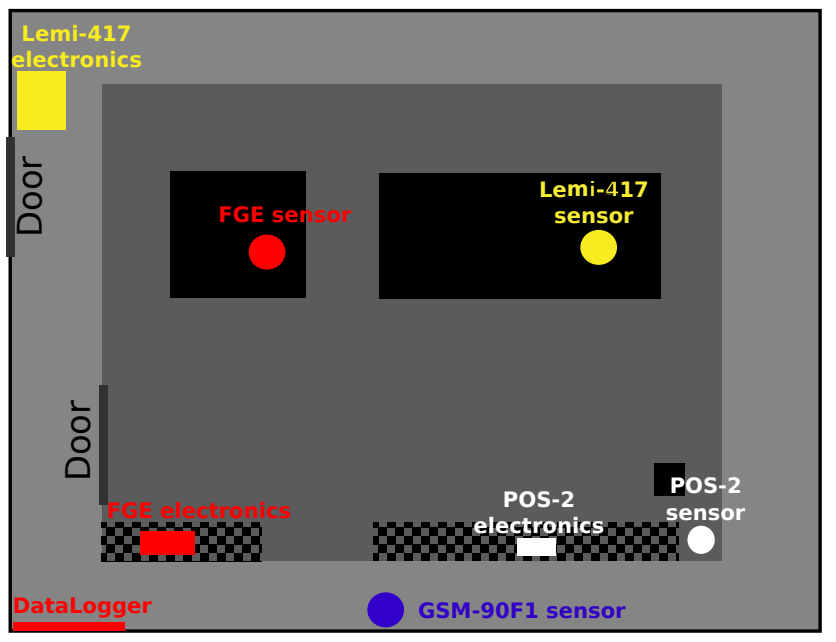

Figure 5. Schematics of the variometer house. The insulated inner room is shown in dark grey. Further, the equipment that has been installed prior to 2015 is shown in white (removed 2015) and yellow (in operation after 2015), the equipment installed in 2015 is shown in red, and the equipment installed in 2016 is shown in blue. Black areas refer to solid stone pillars in the variometer house, black checked areas refer to shelves, and doors are shown in black as well.

tal, charging eight $165 \mathrm{Ah}$ lead-acid batteries, i.e. $1320 \mathrm{Ah}$. In addition, there exist two diesel generators of 5 and $6 \mathrm{~kW}$ at $120 \mathrm{~V}$, which can also be used to charge the batteries. The diesel generator directly powers the lights in the variometer and absolute houses via a dedicated electric cable system. The batteries provide energy mainly for the accommodation building via a $127 \mathrm{~V}$ inverter. In parallel, the batteries power the recently installed equipment (Sect. 4).

\section{Recent improvements}

With the intention to prepare the Tatuoca Observatory to join the INTERMAGNET network, a team of ON and GFZ visited the observatory for two weeks from 17 to 27 November 2015. During this time, new instruments were installed and new methods for absolute measurements were introduced. During a follow-up visit from 24 to 28 October 2016, some further improvements to the instrumentation and absolute measurements were made, as described below.

\subsection{Variometer house}

A Technical University of Denmark (DTU) FGE fluxgate variometer was installed in the variometer house on 21 November 2015 (Pedersen and Merenyi, 2016; Rasmussen and Lauridsen, 1990), and baselines have been available for this variometer since 22 November 2015. As shown in Figs. 5 and 6, the FGE was installed on the existing western socket, at a distance of about $2.2 \mathrm{~m}$ from the LEMI-417 sensor. For testing purposes and as a backup system, the

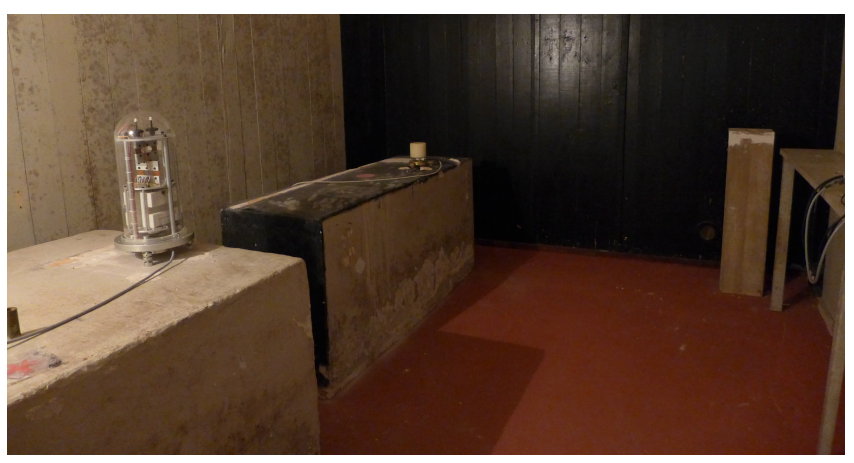

Figure 6. The thermally insulated inner room of the variometer house: the new FGE sensor is placed on the pillar in the front (left), and the LEMI 417 sensor is placed on the pillar in the back. To the right, a part of the shelf with the FGE electronics is visible.

LEMI was kept in operation. The FGE was oriented to magnetic north (HDZ) by minimising the output of its unbiased $Y$-sensor while an appropriate bias field was chosen for the $X$ (horizontal north) and $Z$ (vertical down) channels in order to extend the dynamic range of the readings to the available range of $\pm 10 \mu \mathrm{T}$. The FGE electronics was first placed on the south-eastern shelf and moved to the south-western shelf in October 2016, at a distance of $2.4 \mathrm{~m}$ from the FGE sensor (Fig. 5). At the time of installation, the FGE electronics box was also modified to house a MinGEO ObsDAQ 24 bit analogue to digital converter. Any additional electronic equipment was placed in the south-western corner of the outer corridor (Fig. 5). This equipment consists of a RaspberryPi datalogger system and transformers for powering the FGE and the datalogger. The RaspberryPi has the advantage of low power consumption and easy availability. For more details on the datalogger system, please refer to Morschhauser et al. (2017) in this issue.

Absolute scalar measurements in the variometer house are useful for checking the calibration and resolution of the variometer data. In Tatuoca, a POS-1 and POS-2 were previously installed. However, no consistent readings could be obtained when testing these instruments. Therefore, we have removed the non-operational POS-1 and POS-2 electronics and sensors in November 2015. As a replacement, a GEMSystem GSM-90F1 Overhauser magnetometer was installed in October 2016 (blue symbols in Fig. 5). We used this magnetometer to check that there is no indication of a temperature effect on the variometer by comparing the field intensity readings of both instruments.

\subsection{Power supply}

The newly installed electronics in the variometer house (the FGE fluxgate magnetometer, the GSM-90F1 Overhauser magnetometer, and the datalogging system) are powered by the existing solar cells and batteries which are located in the southern part of the island (Fig. 3). In order to trans- 


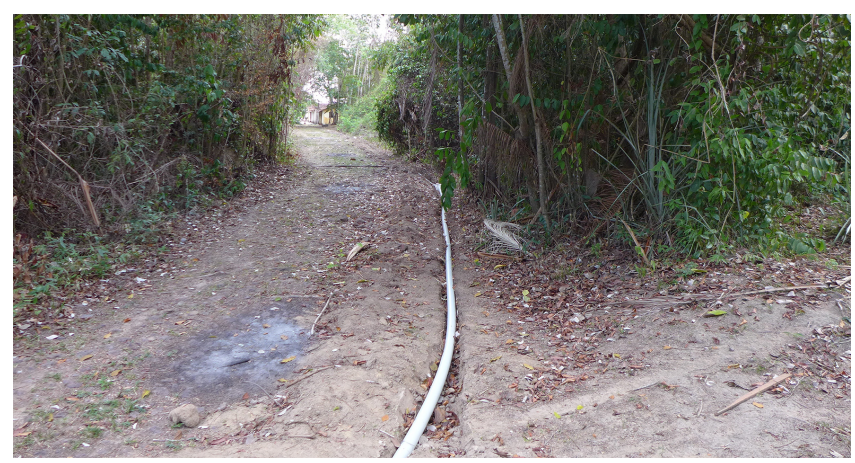

Figure 7. The plastic tube is shown that is used to protect the power supply and fibre optics cable running from the electronics house (visible at the far end in the image) to the variometer house (not visible).

mit power over a distance of $150 \mathrm{~m}$, the $12 \mathrm{~V}$ direct current (DC) of the lead-acid batteries is converted to $220 \mathrm{~V}$ alternate current (AC) using a commercial $300 \mathrm{~W}$ inverter. The power supply line consists of a 3 -wire $1.5 \mathrm{~mm}^{2}$ power cable (H05RN-F 3X1.5) which was installed in a protective plastic tube (Fig. 7). This plastic tube was shallowly buried and can later be used as ductwork for future installations. As lightning occurs frequently near the equator, currents may be induced in the power line by nearby lightning strikes. The currents may easily destroy the sensitive electronics in the variometer house. Therefore, the installation was protected by FURSE (ESP240-16A/BX) overcurrent protectors at each end of the power line. The grounding of these protectors was improved in October 2016 by installing three $2-2.5 \mathrm{~m}$ long copper rods with a diameter of $12 \mathrm{~mm}$ which were connected to the FURSE via $16 \mathrm{~mm}^{2}$ copper cables.

\subsection{Data transmission}

In the same building in which the batteries are located (labelled "electronics" in Fig. 3), a netbook and a 3G router were installed. The netbook can connect to remote servers using a reverse SSH tunnel via the $3 \mathrm{G}$ network. Indeed, increasing coverage by mobile telecommunication network makes data transmission easy and cheap even in more remote places where expensive solutions (satellites, direct link, dedicated landlines) would have been the only alternative before. However, the SIM card that was used to transfer the variometer data stopped working from 4 February to 20 July 2016. Since then, data transfer has been reliable thanks to a new SIM card. The laptop is also used as a backup for the variometer data and displays a daily magnetogram for the local staff to check the correct operation of the system. Since October 2016, the absolute measurements are also manually stored in the netbook and transmitted to a remote server. In this way, quasi-definitive data can be produced with reduced latency. Due to initial problems with a fibre-optical link between the variometer house and the electronics house, the data were manually downloaded from the RaspberryPi datalogger in the variometer house on a daily basis via an ethernet link. Since February 2017, the fibre optics link is fully operational and data are synchronised to a remote server every $15 \mathrm{~min}$. In the future, we plan to implement near-real-time data transfer using a message protocol such as MQTT (Message Queue Telemetry Transport) (Bracke et al., 2017).

\subsection{Absolute measurements}

In the absolute house, changes were kept at a minimum level while making some significant improvements: first, the EDA fluxgate (E.D.A. electronics Ltd., Ottawa, CA) was replaced by a DTU model G fluxgate and electronics (serial number 0151, sensor PIL 7451) on 24 November 2015 after eighteen absolute measurements to determine baselines for the FGE variometer were made.

Second, the absolute house was cleared from a number of magnetic and non-magnetic objects on 26 November 2015. As a result, potential future movement of magnetic objects and associated changes in the level of the observatory (showing up as apparent changes in the baselines) can be avoided Also, a clean absolute house makes it easier to identify new and potentially magnetic objects that have accidentally been forgotten. Before and after removing these objects, five absolute measurements were taken. These 10 absolute measurements revealed a difference in the absolute level of the observatory of $+1.5 \mathrm{nT}$ in the horizontal component $(H)$ and $+0.4^{\prime}(\approx 3 \mathrm{nT})$ in the declination $(D)$ after the removal of these objects while no difference was found for the vertical component. We note that any change in the absolute level should not exceed one $\mathrm{nT}$ in order to preserve the accuracy of the secular variation data from the TTB observatory (Matzka et al., 2010). This could have been achieved by correcting all future or past data with an appropriate constant offset. However, there are strong indications that the absolute level of the observatory was not stable to better than $3 \mathrm{nT}$ in the previous periods, and therefore the previous data have not been corrected for this relatively low change in the observatory's absolute level. Instead, the baseline was adopted by introducing a baseline jump corresponding to the jump in the measured absolute values.

As a consequence of installing the model $\mathrm{G}$ fluxgate, the residual method of absolute measurements was introduced (Jankowsky and Sucksdorff, 1996, p. 89; Worthington and Matzka, 2017, this issue). In this way, the accuracy of the available ZEISS theodolite 020B can be fully used by exactly positioning the horizontal (vertical) circle to full arcminutes during the declination (inclination) measurement. Otherwise, the resolution of the angular readings would have to be estimated to 0.1 arcmin for the $020 \mathrm{~B}$ theodolite. In particular, three pairs of absolute measurements are done per week, and the time is taken from a wall clock set according to the LEMI GPS in the variometer house. However, this clock is magnetic and had to be located far enough from the 


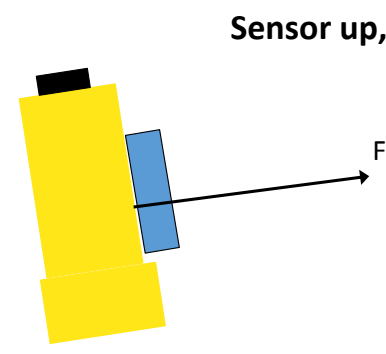

(a) Southern Hemisphere

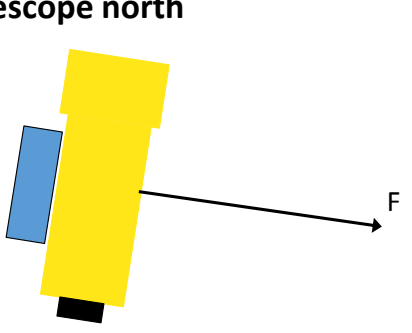

(b) Northern Hemisphere
Figure 8. The position "sensor up, telescope north" is shown for a theodolite near the magnetic equator on the Southern Hemisphere (a) and the Northern Hemisphere (b). Depending on the magnetic hemisphere, these positions differ by $180^{\circ}$.

observer, making it hard to read. Therefore, it was replaced by an almost non-magnetic stopwatch in October 2016. This stopwatch allows the time to be easily read with $1 \mathrm{~s}$ accuracy and is set according to the system time of the netbook in the electronics house. In turn, the netbook's system time is synchronised via NTP with its GPS and several remote NTP servers.

\section{Special considerations for absolute measurements near the magnetic equator}

The standard concepts and observation routines of absolute measurements are challenged at the equator for a number of reasons. Mainly, these challenges result from the trivial fact that inclination is close to zero near the magnetic equator.

A first problem arises as the telescope is nearly vertical during inclination measurements and a zenith ocular is needed to read the vertical circle for positions where the telescope points upwards (for an alternative method, see Brunke and Matzka, 2017). This situation is made even more complicated by the fact that the widely used Zeiss Theo 020B has no degree numbers on the vertical circle from 162 to $179^{\circ}$ and from 181 to $198^{\circ}$. Thus, only the minute marks can be read from the vertical circle if the telescope is pointing down. A slow and cumbersome remedy is to count the number of degree marks between the closest numbered mark and the desired telescope position. Another method is to assume a feasible degree number (e.g. the same one as with the last absolute measurement) and to compare the results of the absolute measurement (baselines, sensor offset, collimation angles) with the previous absolute measurements. In this way, a wrong reading will lead to inconsistent absolute measurements and can easily be identified. Then, the corresponding erroneous reading of the vertical circle must be corrected by a full degree or even multiples of it, and the correct absolute value can be calculated.
Another problem near the magnetic equator arises as formulas to calculate inclination from DI-flux measurements differ in sign for the northern and southern hemispheres (note that Eq. (5.4) of Jankowsky and Sucksdorff, 1996, p. 95 has the wrong sign for the Southern Hemisphere, as well as other sign errors (Matzka and Hansen, 2007)). When the geomagnetic equator is passing the observatory location due to secular variation, it may even happen that an observatory changes its magnetic hemisphere during a single absolute measurement due to the additional daily variation.

Further, telescope positions during inclination measurements are typically denoted "sensor up, telescope north" and so on. If the inclination is very shallow, however, it is not easy to identify whether the telescope actually points south or north, and whether the sensor is positioned up or down relative to the telescope. Here, a simple rule can help to find the correct position: in the Northern Hemisphere, the north-pointing telescope will always point upwards, and in the Southern Hemisphere, the north-pointing telescope will always point downwards (Fig. 8). This still may lead to some confusion if an observatory is changing magnetic hemispheres due to the movement of the magnetic equator. Then, certain positions, e.g. "Sensor up, telescope North" will instantaneously be rotated by 180 degrees (see Fig. 8). However, observers might not realise this situation immediately due to the slow change in inclination and they might report readings in mixed up positions. In this situation, we recommend from our experience that the observers should follow their normal procedure of measurement and any corrections for mixed up positions can be applied during the calculation of baselines.

Still, absolute observations near the magnetic equator do not only make the measurement process more complicated. Since the vertical component is close to zero, the levelling of the telescope is not very critical for declination measurements at the magnetic equator. On the other hand, levelling errors can cause significant problems for observatories at middle to high latitudes, and usually happen due to inexperienced or careless observers.

Although sun observations are not routinely carried out at geomagnetic observatories, they are sometimes necessary for performing accurate absolute measurements as they allow geographical north to be accurately and independently determined. In this case, the standard methods that involve the leading and trailing limb of the sun are not practicable near the geographic equator, where the sun is moving almost vertically. Special considerations on sun observations are detailed in Wienert (1970, p. 136).

\section{Data}

All available digital variometer data of Tatuoca have been processed along with the available absolute measurements. These data include the recordings of the LEMI variometer 


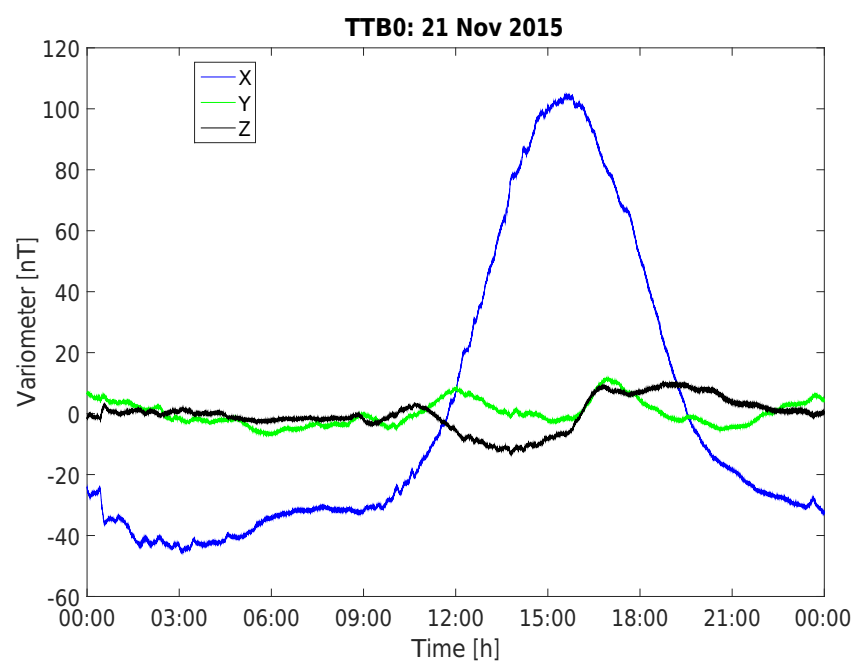

Figure 9. First full day of variometer data of the DTU FGE variometer raw data with $1 \mathrm{~Hz}$ resolution. Time is in UTC. The $X$ sensor is roughly oriented to magnetic north, and the increased amplitude during daytime due to the equatorial electrojet is visible.

from June 2008 to December 2016 and the recordings of the FGE variometer from November 2015 to January 2016. These data will soon be made available at the German Research Centre for Geosciences (GFZ) and the World Data Centre (WDC). Here, we will give a short example of the observed daily variations and present the preliminary base values of the observatory.

On 21 November 2015, the first full day of data was recorded by the DTU FGE variometer. The recorded variations of the $X$ (roughly geomagnetic north), $Y$ (roughly geomagnetic east), and $Z$ (vertical down) components are shown in Fig. 9. On this single day, the signal of the equatorial electrojet (EEJ) is visible as an increase in the $X$-sensor readings during daytime (time in UTC), underlining the importance of the observatory for studying the EEJ.

The preliminary base values of the observatory are shown in Fig. 10 for the horizontal $\left(H_{0}\right)$ field, the declination $\left(D_{0}\right)$, and the vertical field $\left(Z_{0}\right)$. The base values presented here have not been checked for outliers caused by transposed digits or other mistakes in the absolute measurements after January 2016. On the left (Fig. 10a), the base values for the LEMI-417 are shown. Two abrupt changes in the base values (especially $D_{0}$ ) can most likely be attributed to a realignment of the LEMI sensor to geomagnetic north. Further, the base values of the FGE sensor are shown in Fig. 10b, but with a significantly different scaling than for the LEMI sensor. Here, the vertical red lines indicate the period for which direct data transmission from Tatuoca was not possible, and the vertical black lines indicate the beginning and end of the visits to Tatuoca.

The variances of the preliminary base values were estimated by first linearly detrending the data. This detrend-
Table 1. Variances of the base values for the LEMI sensor and the FGE sensor. The variances have been calculated for the horizontal field component $(H)$, declination $(D)$, and the vertical field component $(Z)$ for four different periods.

\begin{tabular}{lcc|ccc}
\hline Instrument: & \multicolumn{2}{c|}{ LEMI } & \multicolumn{3}{c}{ FGE } \\
Period: & I & II & II & III & IV \\
\hline$H_{0}[\mathrm{nT}]$ & 2.74 & 2.40 & 2.29 & 9.44 & 0.76 \\
$D_{0}[\operatorname{arcsec}]$ & 36.60 & 28.36 & 27.55 & 48.62 & 43.43 \\
$Z_{0}[\mathrm{nT}]$ & 3.32 & 3.22 & 1.40 & 3.06 & 2.78 \\
\hline
\end{tabular}

Period I is the time until the first visit (2 June 2008 to 17 November 2015). Period II is the time from after the first visit until the internet connection was lost (28 November 2015 to 3 February 2016.

Period III is the time when internet connection was lost to before the second visit (5 February 2016 to 23 October 2016).

Period IV is the time after the second visit until a lightning strike occurred (29 October 2016 to 30 December 2016).

ing was done separately for periods when the base values changed abruptly. Then, the standard deviation was calculated for different periods and for the horizontal field, the declination, and the vertical field. In Table 1, the resulting standard deviations are summarised. Overall, the base values are stable to within $3 \mathrm{nT}$, but very large outliers occur frequently. For the period before our first visit (period I: 2 June 2008-17 November 2015), only LEMI data are available, and standard deviations are a bit higher compared to period II, which spans the time from after the first visit to before the internet connection was lost (28 November 2015 3 February 2016). Also, the variances of the base values as derived from the FGE sensor are slightly smaller than those of the LEMI sensor, confirming the quality of the FGE instrument. As the internet connection was lost until the second visit (period III: 5 February 2015-23 October 2016), the variances of the base values significantly increased in all three components to a level that would be problematic for an INTERMAGNET observatory. Mainly, the reason is that no immediate feedback could be given to the observatory staff carrying out the absolute measurements, underlining the importance of regular data transmission for ensuring data quality. After our most recent visit in October 2016 (period IV: 29 October 2016 to 30 December 2016), the quality of absolute measurements has improved, although significant scatter still occurs in $\mathrm{D}_{0}$ and $Z_{0}$. However, it is expected that a more detailed investigation and correction or removal of misreadings in the absolute measurements in the course of preparing the definitive data of 2016 will lead to a significantly better standard deviation.

\section{Summary and outlook}

Since 2015, the National Observatory (ON) of Brazil and the German Research Centre for Geosciences (GFZ) have collaborated in preparing the Tatuoca magnetic observatory to become a member of the INTERMAGNET network of mag- 
(a)
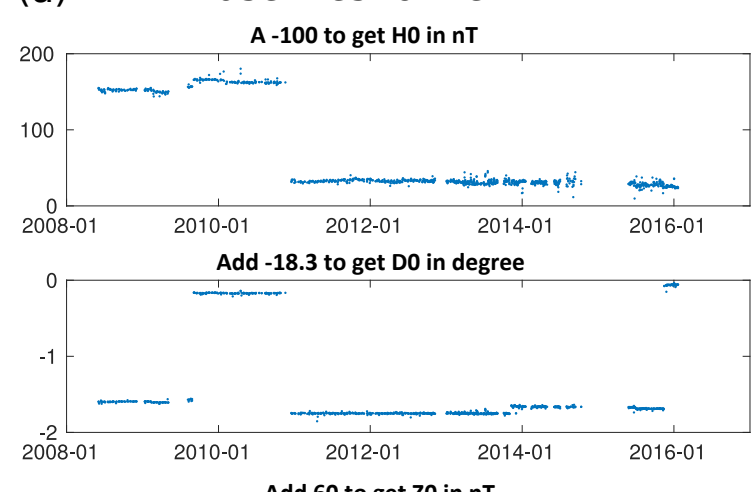

Add 60 to get $\mathrm{ZO}$ in $\mathrm{nT}$

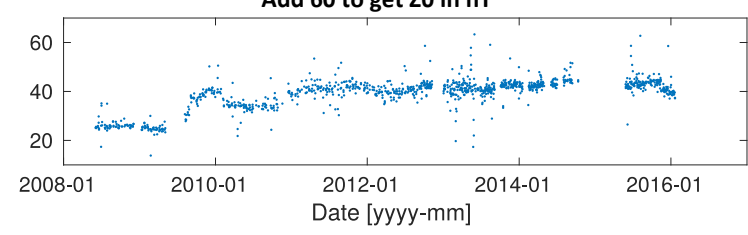

(b) Baselines for DTU FGE
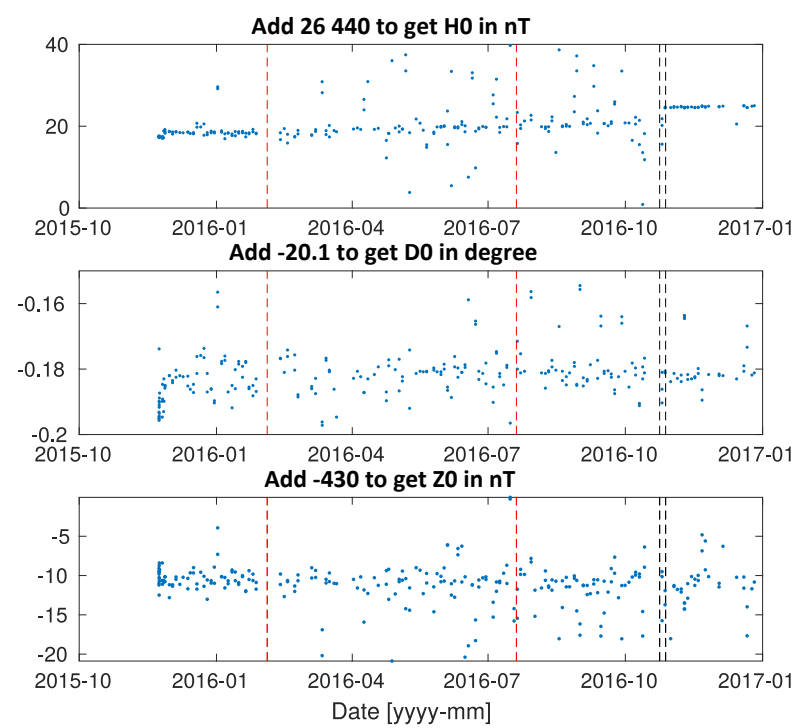

Figure 10. Base values for the Tatuoca observatory are shown for (a) the LEMI-417 variometer, and (b) the DTU FGE variometer. For the period between the vertical red lines, regular data download was not available. Further, vertical black lines indicate the visit to Tatuoca in October 2016.

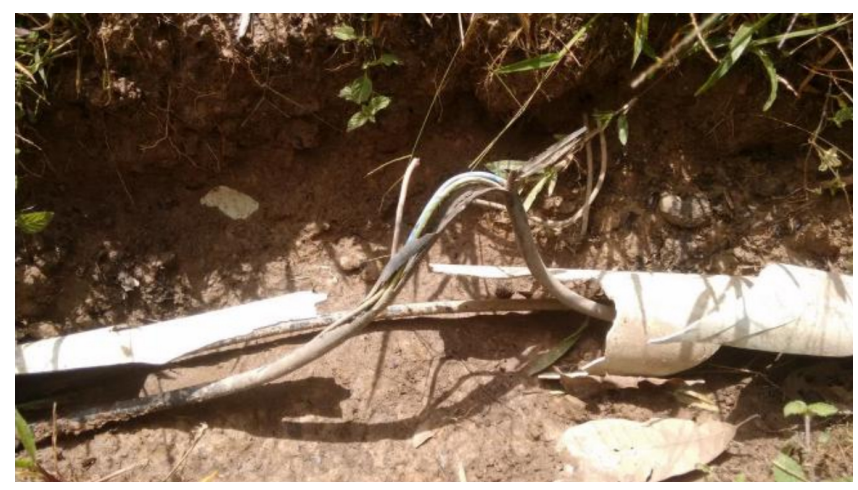

Figure 11. The power cable between the variometer house and the electronics house was damaged a few metres from the variometer house due to currents induced by a lightning strike close to Tatuoca observatory on 31 December 2016.

netic observatories. INTERMAGNET has defined criteria for quality control and data checking, and provides centralised infrastructure for data distribution (Love and Chulliat, 2013). Thus, our efforts will add an observatory adhering to high data quality standards at an interesting location within the magnetic equator and the South Atlantic magnetic anomaly.

As of the end of 2016, a new DTU suspended variometer is installed on Tatuoca along with a modern datalogging system and a GemSystems GSM-90F1 scalar magnetometer. Further, a 3G modem is used to transmit the data to central servers on a daily basis. As well, the EDA fluxgate magnetometer on the ZEISS 020B theodolite in the absolute house was replaced with a DTU fluxgate model G. This lat- ter change allowed the residual method of absolute measurements to be introduced, increasing the accuracy of absolute measurements. Definitive base values have been calculated for the period from 2008 to 2015, and preliminary baselines are available for 2016. Most of the time, the base values are stable to within $3 \mathrm{nt}$, but very large outliers exist. Also, we experienced how important it is to provide immediate feedback to the observatory staff in order to assure high-quality absolute measurements. This is particularly important, as a variety of peculiarities complicate absolute measurements near the equator. For example, missing degree marks at some vertical telescope positions make the readings prone to errors.

On the 31 December 2016, a lightning strike hit the island of Tatuoca. As a consequence, severe currents were induced in the power cable between the variometer house and the electronics house (Fig. 11). Although the installed lightning protection was preventing these currents from destroying the equipment, additional currents were induced in the $10 \mathrm{~m}$ ethernet cable that was attached to the datalogging system. As a consequence, the RaspberryPi was destroyed and the FGE electronics was damaged. Similarly, the inverter, the netbook, and solar charge controllers were destroyed, probably due to induced currents in the cables leading to the batteries and solar panels. This event underlines the importance of lightning protection at magnetic observatories, and we fully repaired the damage in February and March 2017.

Although the observatory is in a promising state, further improvements are required for it to become a reliable member of the INTERMAGNET network. First, the stability of the baseline can still be improved. Second, the power supply 
chain for data recording should become independent from the power supply chain that is available for the housing of the observatory staff.

In addition to these major tasks, there exists various smaller improvements that we may consider in the future. For example, we may include a direct illumination of the theodolite for better readability by using LEDs, and attaching the LEMI variometer to the variometer power supply. The latter will eliminate any potential signal of the DC current that powers the LEMI via solar cells. Also, the temperature stability of the sensor and electronics in the variometer house could be improved by installing an electrical heating and additional insulation around the sensor and electronics.

Data availability. Preliminary magntic field data of the TTB observatory as used in this paper are available from the corresponding author upon request. These data will be distributed in a final version in publicly accessible data repositories.

Author contributions. AM, GBS, JH, JP, KP, and JM were actively participating in the described work and visits to the observatory, OB was programming the datalogger and is responsible for data transmission, AM wrote the article, and $\mathrm{KP}$ and $\mathrm{JM}$ coordinated the activities.

Competing interests. The authors declare that they have no conflict of interest.

Special issue statement. This article is part of the special issue "The Earth's magnetic field: measurements, data, and applications from ground observations (ANGEO/GI inter-journal SI)". It is a result of the XVIIth IAGA Workshop on Geomagnetic Observatory Instruments, Data Acquisition and Processing, Dourbes, Belgium, 4-10 September 2016.

Acknowledgements. We thank Hernández Quintero and one anonymous reviewer for their detailed reviews and suggestions that helped to improve the manuscript. Also, we thank Jean Rasson and the team at the Dourbes Observatory for organising the INTERMAGNET workshop in 2016. Katia Pinheiro acknowledges the support of FAPERJ (Bolsa Jovem Cientista do Nosso EstadoE_06/2015) and Gabriel Brando acknowledges CNPq.

The article processing charges for this open-access

publication were covered by a Research

Centre of the Helmholtz Association.

Edited by: Jean Rasson

Reviewed by: Esteban Hernandez-Quintero and one anonymous referee

\section{References}

AGU: New geophysical observatory, Tatuoca, Belem, Brazil, J. Geophys. Res., 60, 117-117, https://doi.org/10.1029/jz060i001p00117-01, 1955.

Bracke, S., Gonsette, A., Rasson, J., Poncelet, A., and Hendrickx, O.: Automated observatory in Antarctica: real-time data transfer on constrained networks in practice, Geosci. Instrum. Method. Data Syst. Discuss., https://doi.org/10.5194/gi-2017-17, in review, 2017.

Brunke, H.-P. and Matzka, J.: Numerical Evaluation of magnetic absolute Measurements with arbitrary distributed DI-Fluxgate Theodolite Positions, Geosci. Instrum. Method. Data Syst. Discuss., https://doi.org/10.5194/gi-2017-3, in review, 2017.

Buchanan, A., Finn, C. A., Love, J. J., Worthington, E. W., Lawson, F., Maus, S., Okewunmi, S., and Poedjono, B.: Geomagnetic Referencing - The Real-Time Compass for Directional Drillers, Oilfield Review, 25, 32-47, 2013.

Ferreira, J. T.: Resultados Magnéticos de Tatuoca - 1989, Publicaçõ Especial do Observatório Nacional, 1990.

Ferreira, J. T.: Resultados Magnéticos de Tatuoca - 1997, Publicaçõ Especial do Observatório Nacional, 1998.

Gama, L. I.: Installation of the Tatuoca Magnetic Observatory, vol. 6, Publicações do Serviço Magnético, 1955.

Hulot, G., Sabaka, T., Olsen, N., and Fournier, A.: The Present and Future Geomagnetic Field, in: Treatise on Geophysics, Elsevier BV, 5, 33-78, https://doi.org/10.1016/b978-0-444-538024.00096-8, 2015.

IUGG, IPY: Geomagnetic K-indices, Assoc. Terr. Mag. Electr., Bull. No. 12d, 1950.

Jankowsky, J. and Sucksdorff, C.: Guide for magnetic measurements and observatory practice, IAGA, International Association of Geomagnetism and Aeronomy (IAGA), ISBN: 10-9650686-2$5,1996$.

Love, J. J. and Chulliat, A.: An International Network of Magnetic Observatories, EOS, 94, 373-374, https://doi.org/10.1002/2013eo420001, 2013.

Manoj, C., Lühr, H., Maus, S., and Nagarajan, N.: Evidence for short spatial correlation lengths of the noontime equatorial electrojet inferred from a comparison of satellite and ground magnetic data, J. Geophys. Res., 111, A11312, https://doi.org/10.1029/2006JA011855, 2006.

Matzka, J. and Hansen, T. L.: On the Various Published Formulas to Determine Sensor Offset and Sensor Misalignment for the DIflux, Publ. Inst. Geophys. Pol. Acad. Sci., C-99, 152-157, 2007.

Matzka, J., Chulliat, A., Mandea, M., Finlay, C. C., and Qamili, E.: Geomagnetic Observations for Main Field Studies: From Ground to Space, Space Sci. Rev., 155, 29-64, https://doi.org/10.1007/s11214-010-9693-4, 2010.

Morschhauser, A., Haseloff, J., Bronkalla, O., MüllerBrettschneider, C., and Matzka, J.: A low-power data acquisition system for geomagnetic observatories and variometer stations, Geosci. Instrum. Method. Data Syst. Discuss., https://doi.org/10.5194/gi-2017-23, in review, 2017.

Newitt, L. R., Barton, C. E., and Bitterly, J.: Guide for Magnetic Repeat Station Surveys, IAGA, International Association of Geomagnetism and Aeronomy (IAGA), ISBN 10-9650686-17, 1996. 
Nicolet, M. and Doyen, P.: Geopgraphical Distribution of the International Geophysical Year Stations, in: Annals of the International Geophysical Year, vol. VIII, Paergamon Press Ltd., 1959.

Olsen, J.: Results of magnetic observations made at Tatuoca (Brazil), September 1933-January 1934, Temporary Commission on the Liquidation of the Polar Year (TCLPY) 1932-33, International Meteorological Organization, 1951.

Pedersen, L. W. and Merenyi, L.: The FGE Magnetometer and the INTERMAGNET 1 Second Standard, Journal of the Indian Geophysical Union, 2, 30-36, 2016.

Rasmussen, O. and Lauridsen, E.: Improving baseline drift in fluxgate magnetometers caused by foundation movements, using band suspended fluxgate sensors, Phys. Earth Planet. In., 59, 78 81, https://doi.org/10.1016/0031-9201(90)90211-f, 1990.
Thébault, E., Finlay, C. C., Beggan, C. D., Alken, P., Aubert, J., Barrois, O., Bertrand, F., Bondar, T., Boness, A., Brocco, L., Canet, E., Chambodut, A., Chulliat, A., Coïsson, P., Civet, F., Du, A., Fournier, A., Fratter, I., Gillet, N., Hamilton, B., Hamoudi, M., Hulot, G., Jager, T., Korte, M., Kuang, W., Lalanne, X., Langlais, B.; Léger, J.-M., Lesur, V., Lowes, F. J., Macmillan, S., Mandea, M., Manoj, C., Maus, S., Olsen, N., Petrov, V., Ridley, V., Rother, M., Sabaka, T. J., Saturnino, D., Schachtschneider, R., Sirol, O., Tangborn, A., and Thomson, A., Tøffner-Clausen, L., Vigneron, P., Wardinski, I., and Zvereva, T.: International Geomagnetic Reference Field: the 12th generation, Earth Planet. Space, 67, 217 pp., https://doi.org/10.1186/s40623-015-0228-9, 2015.

Wienert, K. A.: Notes on geomagnetic observatory and survey practice, Earth Sciences, UNESCO, 5, p. 79, 1970.

Worthington, E. W. and Matzka, J.: USGS Experience with the Residual Absolutes Method, Geosci. Instrum. Method. Data Syst. Discuss., https://doi.org/10.5194/gi-2017-24, in review, 2017. 